\title{
PERCEPÇÃO DOS USUÁRIOS DE BOMBA DE INFUSÃO DE INSULINA NO SUDESTE GOIANO
}

\author{
A. C. SILVA , A. M. SILVA, A. O. S. M. SILVEIRA, S. SOUZA, G. S. LEAL, M. R. BARROS \\ Universidade Federal de Goiás - Regional Catalão-GO \\ ancarsil@ufg.br \\ Submetido 30/03/2017 - Aceito 21/09/2017 \\ DOI: $10.15628 /$ holos. 2017.4818
}

\section{RESUMO}

A partir do final do século $X X$, surgiram grandes avanços tecnológicos no tratamento do diabetes, entre eles destaca-se a Bomba de Infusão de Insulina (BII). Diante da busca de tratamentos que alcancem um controle glicêmico adequado passível de prevenir as complicações crônicas e com melhor qualidade de vida, percebeu-se a necessidade de avaliar a satisfação com o tratamento (ST) BII. A amostra selecionada foi composta por 10 indivíduos de um total de 12 usuários de Bll que responderam um questionário sobre ST. A maioria dos pacientes entrevistados apresentou alto percentual em relação ao grau de satisfação com a BII. Por outro lado, os altos custos e a distorção da imagem corporal destacaram-se como fatores negativos em relação a Bll. Apesar da Bll ser uma inovação tecnológica no tratamento de pacientes diabéticos, ainda é subutilizada principalmente pelos custos elevados. Dessa forma é fundamental a realização de mais pesquisas na área, para que seja possível demonstrar custo-benefício do tratamento com a BII, possibilitando maior abrangência dessa terapia para a população diabética, assim como disponibilização de dispositivos mais confortáveis.

\section{PERCEPTION OF USERS OF INSULIN INFUSION PUMP IN SOUTHEAST GOIANO}

\begin{abstract}
From the end of the twentieth century brought major technological advances in the treatment of diabetes, among them stands out the Insulin Infusion Pump (BII). On the search for treatments that achieve a proper glycemic control capable of preventing chronic and better quality of life complications, we realized the need to assess satisfaction with treatment (ST) BII. The selected sample consisted of 10 individuals from a total of 12 users Bll who answered a questionnaire about ST. Most patients interviewed showed a high percentage in relation to the degree of satisfaction
\end{abstract}

with BII. On the other hand, the high costs and distorted body image stood out as negative factors in relation to BII. Despite the BII be a technological breakthrough in the treatment of diabetic patients, it is still underutilized mainly by high costs. Thus it is essential to further research in the area, so that you can demonstrate cost-effectiveness of treatment with BII, allowing greater scope of this therapy for diabetic population, as well as providing more comfortable devices.

KEYWORDS: diabetes mellitus type 1, pump of infusion of insulin, satisfaction 


\section{INTRODUÇÃO}

O tratamento do Diabetes Mellitus (DM) Tipo 1 tem passado por avanços tecnológicos nas últimas décadas. A administração de insulina tem sido a única forma de tratamento disponível para milhões de pacientes com esta doença a nível mundial.

Após a descoberta da insulina, em 1921, a sua administração começou a ser realizada por via intramuscular, marcada por dor intensa no local da aplicação. Desde então tem evoluído as formas de aplicação de insulina permitindo maior comodidade e conforto, como a terapia com uso de seringas e canetas de uso subcutâneo. A busca de novas formas de aplicação de insulina tem se intensificado nos últimos anos, com ênfase na utilização da Bomba de Infusão de Insulina (Mala \& Araújo, 2003).

Por se tratar de uma doença de natureza crônica, a gravidade das complicações e os meios utilizados para seu controle, tornam o DM uma enfermidade muito onerosa não somente para os indivíduos afetados e suas famílias, mas também para o sistema de saúde. Por isso é de suma importância buscar tratamentos que alcancem um controle glicêmico adequado, prevenindo o desenvolvimento de complicações crônicas da doença e com melhoria na qualidade de vida (QV).

Diante da evolução tecnológica no tratamento do DM, a qual proporciona alternativas terapêuticas que visam maior conforto e comodidade, percebeu-se a necessidade de avaliar a satisfação com o tratamento Bomba de Infusão de Insulina (BII), também denominado Sistema de Infusão Contínua de Insulina ( $\mathrm{SICl}$ ) em pacientes portadores de DM1 assistidos em uma Associação de Diabéticos do Sudeste Goiano (ADISGO).

A ADISGO é uma entidade fundada em 25 de outubro de 1990, na cidade de Catalão, município brasileiro do estado de Goiás, cuja população segundo estimativas do Instituto Brasileiro de Geografia e Estatística (IBGE) 2015, é de 98737 habitantes. Essa instituição não tem fins lucrativos e possui a finalidade de prestar serviços gratuitamente a população. Atualmente consta com mais de 3700 associados, além de uma equipe multidisciplinar composta por educadores em diabetes, nutricionista, podólogo, psicólogo e médicos.

\section{REFERENCIAL TEÓRICO}

\subsection{Diabetes Mellitus}

O Diabetes Mellitus representa um grupo de doenças metabólicas, com etiologias diversas, caracterizados pela hiperglicemia, que resulta de uma secreção deficiente de insulina pela célula beta, resistência periférica à ação da insulina ou ambas. A classificação atual segundo a Associação Americana de Diabetes (ADA) inclui 4 classes clínicas: DM tipo 1 (DM1), DM tipo 2 (DM2), outros tipos específicos de DM e DM Gestacional, conforme quadro 1: 


\section{Quadro 1: Classificação etiológica do DM}

\begin{tabular}{|c|l|}
\hline Classificação etiológica do DM & \multicolumn{1}{|c|}{ Características } \\
\hline DM 1 & $\begin{array}{l}\text { Representa } 5 \text { a } 10 \% \text { dos casos de diabetes; } \\
\text { Resulta da destruição das células betapancreáticas } \\
\text { com deficiência da secreção de insulina; } \\
\text { Ocorre com maior frequência em crianças e } \\
\text { adolescentes, mas pode ocorrer em adultos. }\end{array}$ \\
\hline DM 2 & $\begin{array}{l}\text { Representa 90 a 95\% dos casos de diabetes; } \\
\text { Resulta de defeitos na ação e secreção da } \\
\text { insulina; } \\
\text { Pode ocorrer em qualquer idade, mas é } \\
\text { geralmente diagnosticado após os 40 anos; } \\
\text { A maioria dos pacientes com essa forma de } \\
\text { diabetes apresenta sobrepeso ou obesidade. }\end{array}$ \\
\hline DM Gestacional & $\begin{array}{l}\text { Ocorre em 1 a 14\% de todas as gestações, } \\
\text { dependendo da população estudada; } \\
\text { Trata-se de qualquer intolerância à glicose } \\
\text { com início ou diagnóstico durante a gestação. }\end{array}$ \\
\hline $\begin{array}{l}\text { Corresponde aos defeitos genéticos na função } \\
\text { das células betas e na ação da insulina; } \\
\text { doenças do pâncreas exócrino; } \\
\text { endocrinopatias; induzido por medicamentos } \\
\text { ou agentes químicos; infecções e outra } \\
\text { síndromes genéticas. }\end{array}$ \\
\hline
\end{tabular}

Segundo dados da Federação Internacional de Diabetes (IDF, 2015), uma epidemia de Diabetes Mellitus está em curso. Atualmente estima-se mundialmente que 415 milhões de adultos estejam afetados pela doença, com previsão para alcançar 642 milhões em 2040 e que 542.000 crianças têm DM1. A maioria desses indivíduos residem em países em desenvolvimento, onde a epidemia tem maior impacto e há crescente proporção de pessoas afetadas em grupos etários mais jovens.

No final da década de 1980, estimou-se no Brasil a prevalência de DM na população adulta em 7,6\% (Malerbi \& Franco, 1992), e dados mais recentes mostram aumento crescente da prevalência. Estudo recente realizado, em seis capitais brasileiras, com servidores de universidades públicas, na faixa etária de 35 a 74 anos, demonstrou uma prevalência de cerca de $20 \%$, sendo que metade dos casos desconhecia a doença no momento do diagnóstico (Schmidt, et al., 2014). 
A incidência de DM1 evidência acentuada variação geográfica, apresentando taxas por 100 mil indivíduos com menos de 15 anos de idade: 38,4 na Finlândia, 7,6 no Brasil, e 0,5 na Coréia. Recentemente, evidenciou-se que a incidência de DM1 tem aumentado, principalmente na população infantil com menos de 5 anos de idade (Onkamo, Karvonen \& Tuomilehto, 1999). Segundo IDF (2015) existem no Brasil 30.900 indivíduos na faixa etária de 0-14 anos acometidos pela doença. Por outro lado, avaliar a incidência de portadores de Diabetes Tipo 2 em grandes populações torna-se difícil, pois requer seguimento por alguns anos, com avaliações de medições periódicas das glicemias.

\subsection{Bomba de infusão de insulina}

A primeira bomba de infusão de insulina surgiu em 1963, foi desenvolvida por Kadish, nos Estados Unidos da América. Tratava-se de uma bomba enorme amarrada ao dorso do paciente, semelhante a mochila usada atualmente pelos adolescentes, a qual realizava infusão endovenosa de insulina e glucagon (apud Mala \& Araújo, 2003).

Entre os mais importantes avanços tecnológicos que possibilitaram o gerenciamento eletrônico do DM, destaca-se o uso do Sistema de Infusão Contínua de Insulina. Embora tenha sido desenvolvida nos EUA desde 1970, os SICl começaram a serem usados em estudos como o Diabetes Control and Complications Trial (DCCT, 1993), onde demonstrou-se que o controle rígido dos níveis glicêmicos reduz os riscos de retinopatia, nefropatia e neuropatia.

No Brasil chegou há cerca de 15 anos. De acordo com o primeiro simpósio internacional de novas tecnologias em diabetes (SITEC, 2015), demonstrou-se dados de uma pesquisa comparativa realizada com apoio da SBD, do uso de BII na América do Sul que apontou entre 4 a 5 mil pacientes utilizam o equipamento no Brasil , para uma população de 200 milhões de habitantes. Por outro lado, dados americanos, referenciaram que 600 mil pessoas usam a Bll para uma população aproximadamente de 312 milhões de habitantes.

O SICl é um dispositivo mecânico com comando eletrônico, do tamanho de um celular, pesando de 80 a 100 gramas, sendo aderida externamente ao corpo através de uma cânula flexível de teflon, que tem uma agulha-guia, a qual é inserida sobre a pele principalmente no abdômen, e por ela envia insulina no tecido subcutâneo do paciente. A liberação de insulina durante $24 \mathrm{~h}$ é automática e feita por meio de uma programação prévia, podendo ser constante ou variável. Esses aparelhos simulam o que acontece na fisiologia normal, com liberação contínua de insulina (basal) e por meio de pulsos (bolus) nos horários das refeições, ou para corrigir a hiperglicemia, sendo capazes de fornecer flexibilidade ao estilo de vida, particularmente em relação aos horários de refeições e viagens (Minicucci, Figueiredo, Araújo, \& Pimazoni-Netto, 2007).

Atualmente no Brasil, dispõe-se de bombas de infusão de dois fabricantes, o laboratório Roche e a empresa de medicamentos Medtronic. O Roche comercializa as Bombas AccuCheckSpirit e o Sistema Accu-CheckSpirit Combo, e a Medtronic disponibiliza os modelos Paradigm 722 e Veo. 
A bomba de infusão paradigma VEO está disponível no Brasil desde 2014, a qual acoplada a um sensor de glicose da Paradigma 722, interrompe automaticamente a infusão de insulina, o que em caso de hipoglicemia severa pode impedir o coma e a morte por hipoglicemia (SBD, 2016).

O quadro 2 mostra as características quanto ao uso da bomba de infusão através da ferramenta matriz SWOT, cujo termo é um acrônimo das palavras strenghts, weakenesses, opportunites e threats que significam respectivamente: forças, fraquezas, oportunidades e ameaças.

Quadro 2: Análise SWOT da bomba de infusão de insulina

\begin{tabular}{|c|c|}
\hline \multirow{4}{*}{ BOMBA DE INFUSÃO DE INSULINA } & $\begin{array}{l}\text { Vantagens (Pontos fortes) } \\
\text { Elimina a necessidade de várias aplicações de } \\
\text { insulina durante o dia; } \\
\text { Libera as doses necessárias com mais exatidão do } \\
\text { que as injeções; } \\
\text { Resulta em variações menores na oscilação } \\
\text { glicêmica; } \\
\text { Redução dos episódios de hipoglicemia grave; } \\
\text { Melhoria da qualidade de vida. }\end{array}$ \\
\hline & $\begin{array}{l}\text { Desvantagens (Pontos fracos) } \\
\text { Custo mais elevado entre as opções disponíveis de } \\
\text { insulinoterapia; } \\
\text { Necessita treinamento especializado; } \\
\text { Desconforto com o cateter e com a bomba de } \\
\text { insulina conectada ao corpo. }\end{array}$ \\
\hline & $\begin{array}{l}\text { Ameaças } \\
\text { Pode desencadear cetoacidose diabética, caso o } \\
\text { cateter seja desconectado ou obstruído por tempo } \\
\text { prolongado; } \\
\text { Infecções de pele; } \\
\text { Falhas das bombas. }\end{array}$ \\
\hline & $\begin{array}{l}\text { Oportunidades } \\
\text { Proporciona ao usuário maior flexibilidade em } \\
\text { relação à dieta; } \\
\text { Melhoria no controle do exercício físico; } \\
\text { Promove maior facilidade com turnos de trabalhos } \\
\text { e viagens. }\end{array}$ \\
\hline
\end{tabular}

Os pontos fracos apontados no quadro 2 são corroborados por frases obtidas através de depoimentos com os usuários de BII, quando são questionados sobre o que mais lhe desagradam em relação ao uso da BII, destaca-se: "O desconforto que está instalada no seu corpo. " / "Talvez o 
tamanho poderia ser um pouco menor". Já os pontos fortes são explícitos quando um entrevistado descreve sobre o que mais lhe agrada na BII: "A independência e a facilidade de levar uma vida praticamente normal. "

Atualmente as principais indicações para o uso da BII no DM tipo 1, refere-se ao controle inadequado, hipoglicemias graves, recorrentes, assintomáticas, ocorrência do fenômeno de alvorecer ou entardecer, necessidade de maior flexibilidade no estilo de vida, gastroparesia, cetoacidoses recorrentes e mulheres que desejam engravidar (SBD, 2016). Por outro lado, a Associação Americana da Diabetes (ADA) sugere que todos os pacientes motivados e com desejo de assumir responsabilidade por seu autocontrole são candidatos ao uso da Bll.

O grande obstáculo dessa forma de terapia insulínica tem sido o alto custo. De acordo com a consultora do Laboratório Roche em Goiás, o preço do equipamento em 2016 está em torno de $R \$ 13.800,00$ e a manutenção mensal cerca de $R \$ 1.000,00$, sendo o custo mais alto quando comparado ao tratamento com múltiplas doses de insulinas (MDI) diárias.

Assim sendo, esta decisão deve ser individualizada levando em consideração a preferência do doente. Refere-se a uma modalidade terapêutica exigente a nível pessoal, requerendo uma grande motivação para seu tratamento, cooperação com os profissionais de saúde, compreensão da tecnologia e capacidade para utilização da bomba e automonitorização frequente da glicemia. É fundamental uma equipe multidisciplinar que tenha um programa educacional estruturado sobre o cálculo de carboidratos e treino do uso de bolus de correção.

No contexto percebe-se que o $\mathrm{SICl}$ é uma inovação tecnológica que permite a melhoria da QV nos pacientes portadores de DM1. A avaliação da satisfação com o tratamento (ST), tem interesse particular no DM devido à complexidade do tratamento bem como as suas influências na qualidade de vida. Evidenciou-se que a ST afeta o comportamento do doente em relação a sua saúde, consequentemente tem impacto nos resultados da terapia. A avaliação da satisfação fornece um meio de quantificar o efeito do tratamento nos doentes, permitindo a possibilidade de desenvolver métodos de tratamento com melhor aceitação (Brod, Hristensen \& Bushnell, 2007).

\subsection{Disponibilidade da bomba de insulina}

No Brasil as bombas de infusão ainda não são disponibilizadas pelo sistema único de saúde e nem pelos planos de saúde. A maioria delas são fornecidas através de requisições por ações judiciais contra o estado, município e governo federal. Todavia alguns estados brasileiros já possuem protocolos clínicos para dispensarem o sistema de infusão contínua de insulina sem a necessidade dos solicitantes recorrerem ao setor judiciário.

Nesse contexto, destaca-se Goiânia que desde 12/08/2011 publicou em Diário Oficial do Município a Portaria 218, definida pela Secretaria Municipal de Saúde de Goiânia, liberando o SICI assim como seus insumos para manutenção do tratamento, aos pacientes residentes em Goiânia, que preenchem os critérios clínicos definidos em protocolo.

Em Catalão - GO, as bombas de insulina foram disponibilizadas da seguinte forma: 4 doadas por um dos laboratórios fabricantes, 3 por recursos próprios do paciente e 3 por ações 
judiciais ( 2 contra o município e 1 contra o estado). Porém, os insumos utilizados para a manutenção mensal do aparelho foram adquiridos e são mantidos pela Secretaria Municipal de Saúde, através de parcerias com a ADISGO.

\section{METODOLOGIA}

Trata-se de um estudo de abordagem quantitativa, transversal e descritiva. De acordo com Flick (2013) esse modelo de pesquisa apresenta uma descrição numérica de uma população que possibilita uma análise estatística dos dados.

O estudo foi realizado em uma Associação de Diabéticos do Sudeste Goiano (ADISGO), no município de Catalão - GO, no período de 18 a 20 de março de 2017. A escolha dessa instituição como cenário de pesquisa, se deu pelo fato dos pacientes procurarem a entidade para serem assistidos durante a instalação do $\mathrm{SICl}$ e/ou posteriormente para orientações ao longo do tratamento.

Participaram da pesquisa 10 pacientes usuários de Bll de um total de 12 indivíduos que utilizam a BlI no município. Todos os participantes assinaram o termo de consentimento livre e esclarecido, sendo garantido o caráter confidencial e voluntário da participação na pesquisa, sendo esta aprovada pelo comitê de ética sob parecer número 1.963.714.

A coleta de dados foi realizada através de um instrumento proposto por Apolinário, Silva \& Cardoso (2011). Esse questionário é composto por 46 questões. As primeiras 30 questões são relacionadas com o grau de satisfação com o uso da bomba de infusão. As questões 31 a 34 referem-se a domínios em que o próprio entrevistado pontua sobre a satisfação ou insatisfação no tratamento com a mesma. As opiniões dos pacientes de situações que agradam e desagradam com o tratamento foram tratadas nas questões 43 e 44 . A indicação da recomendação do uso ou não da Bll é tratada no item 45 . O questionário é finalizado com a questão de número 46 onde fazse uma comparação com o tratamento previamente realizado a colocação da BII.

Foi aplicado paralelamente ao instrumento anterior, outro questionário relacionado com o perfil sóciodemográfico constituído por cinco questões sobre: sexo, idade, escolaridade, estado civil e situação profissional atual. Pesquisou-se ainda a data de colocação da bomba, duração do tempo de doença e o motivo da colocação do SICl.

\section{RESULTADOS}

Percebe-se que houve uniformidade no que tange estado civil e escolaridade. A amostra foi bem distribuída, com idades compreendidas entre 14 e 43 anos. Em relação ao tratamento de DM, o tempo de uso de Bll variou entre 1 a 5 anos (40\%), de 6 a 10 anos (40\%) e de 11 a 15 anos (20\%), a média geral do tempo de uso da bomba é de 6,1 anos e a média de tempo de duração da doença foi de 17,6 anos. Os resultados do questionário sóciodemográfico são apontados na Figura 1 abaixo: 


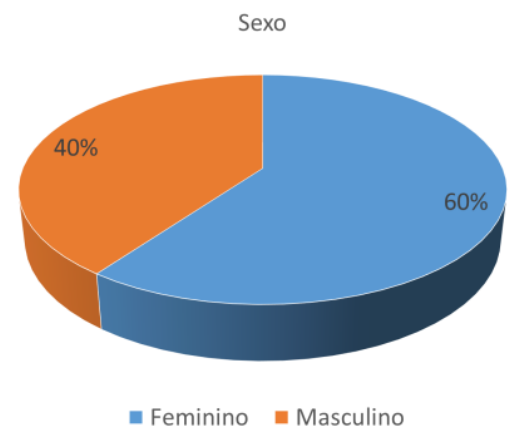

(a)

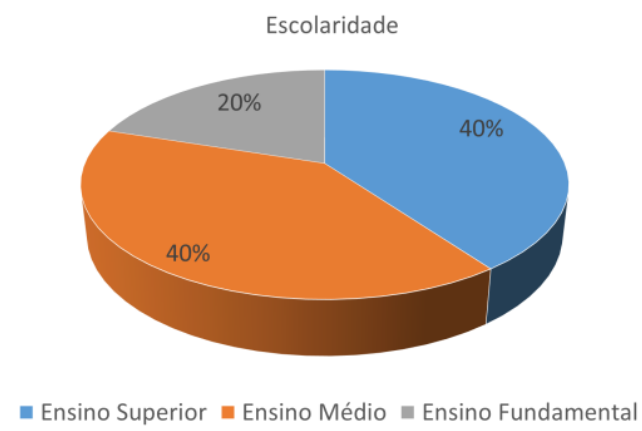

(c)

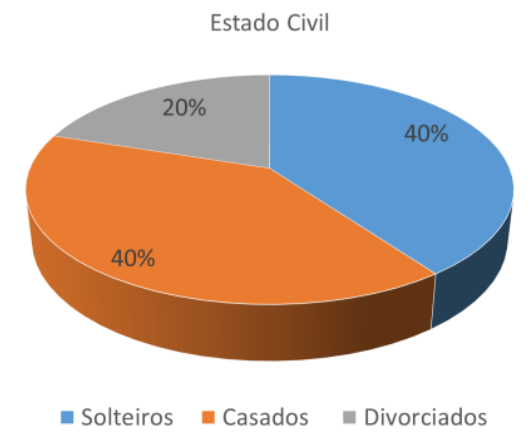

(b)

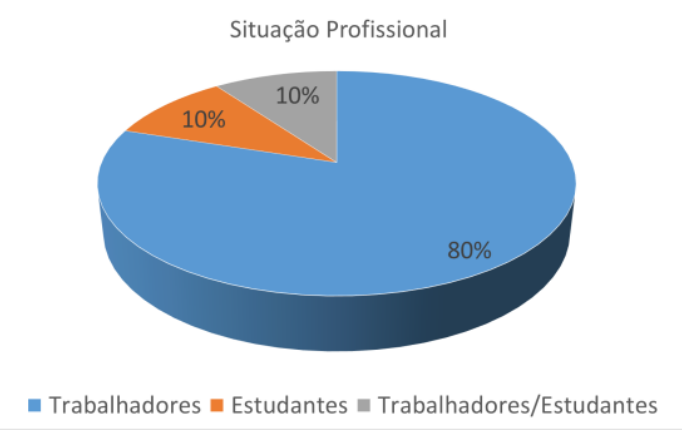

(d)

Figura 1. Dados sóciodemográficos de acordo com: (a) Sexo; (b) Estado Civil; (c) Escolaridade e (d) Situação Profissional.

A maioria das respostas apresentou um alto percentual de satisfação ao uso da Bll. Porém, quando se analisa os custos econômicos com o tratamento, o índice de insatisfeitos (33,3\%) somados aos de nem satisfeitos ou nem insatisfeitos (11.1\%) somou-se um percentual de $44 \%$. Esses dados corroboram com Liberatore \& Damiani (2006) onde demonstrou-se que os custos econômicos são elevados para a aquisição da Bll, quanto para os insumos necessários para sua utilização. Os principais motivos para a colocação da Bll são apresentados na Tabela 1, predominando o quesito hipoglicemia.

Tabela 1: Indicação para a colocação da bomba

\begin{tabular}{|c|c|}
\hline Indicação & Percentual \\
\hline Hipoglicemia & 50 \\
\hline Controle glicêmico instável & 20 \\
\hline Qualidade de vida & 20 \\
\hline Flexibilidade no estilo de vida & 10 \\
\hline
\end{tabular}

Em relação ao desconforto associado com o tratamento (dor, transporte do aparelho, uso de adesivos) o índice de insatisfeito (10\%) somado ao nem satisfeito ou nem insatisfeito (20\%) 
somou-se 30\%. A Tabela 2 aponta os principais fatores que agradam e desagradam os entrevistados em relação ao uso da BII:

Tabela 2: O que agrada e o que desagrada do uso da BII

\begin{tabular}{|c|c|c|c|}
\hline O que agrada & Percentual & O que desagrada & Percentual \\
\hline Controle glicêmico & 50 & Incômodo do aparelho ligado ao corpo & 30 \\
\hline Qualidade de vida & 30 & Nada desagrada o usuário & 30 \\
\hline Flexibilidade no estilo de vida & 10 & Preencher o cartucho & 10 \\
\hline Diminuição do no de picadas & 10 & Tamanho da bomba & 10 \\
\hline \multirow{2}{*}{} & & Desconforto com o cateter & 10 \\
\cline { 3 - 4 } & & Durabilidade & 10 \\
\cline { 3 - 4 }
\end{tabular}

Na questão em que se trata o que mais lhe agrada no tratamento com BII, o controle glicêmico e a qualidade de vida somaram $80 \%$ das respostas. Por outro lado, o questionamento que mais lhe desagrada no tratamento com BII, foi o incômodo de manter o aparelho conectado ao corpo (30\%) e o desconforto com o cateter (10\%), totalizando $40 \%$. Segundo as Diretrizes da Sociedade Brasileira de Diabetes (2016), a distorção da imagem corporal pelo aparelho situa-se entra uma das causas de abandono e descontinuidade do uso da BII.

A satisfação dos pacientes com Bll foi avaliada do item 01 a 30, sendo a maioria como satisfeitos ou muito satisfeitos. As demais questões não foram incluídas na análise porque a amostra foi insuficiente.

Por fim, todos recomendaram o uso da Bll e observou-se que o tratamento com o $\mathrm{SICl}$ é melhor que a terapia anterior utilizada com múltiplas doses diárias de insulina.

\section{CONCLUSÕES}

A bomba de infusão de insulina tem se mostrado como método de terapia de reposição de insulina para pacientes diabéticos de forma eficaz, com resposta clínica satisfatória, maior comodidade, precisão de dose, menos riscos de hipoglicemias, menores oscilações glicêmicas, melhor qualidade de vida, maior adesão ao tratamento e segurança para os pacientes.

Neste estudo observou-se através do questionário aplicado, um alto índice de satisfação em relação com o tratamento Bll. Todavia, os altos custos econômicos com a terapia e a distorção da imagem corporal foram os quesitos pontuados com impacto negativo relacionados ao uso da BII.

Embora o SICl seja um recurso tecnológico altamente eficiente, ainda é subutilizado, devido ao alto custo do tratamento e por depender da capacidade de entendimento, do nível de educação em diabetes do paciente e da equipe multidisciplinar. Além desses fatores evidenciou-se como obstáculos ao uso dessa terapia, o desconforto e a distorção da imagem corporal de ser ter um aparelho conectado ao corpo. 
Finalmente, a partir dos dados apresentados, ressalta-se a necessidade de maior divulgação do $\mathrm{SICl}$ para os pacientes, assim como treinamentos através de educação médica continuada para endocrinologistas que se propõem a tratarem DM1. Além disso, é fundamental a realização de mais pesquisas na área, para que seja possível demonstrar custo-benefício do tratamento com BII, possibilitando maior abrangência dessa terapia para a população diabética, assim como a disponibilização de $\mathrm{SICl}$ mais confortáveis e menores para serem acoplados ao corpo.

\section{AGRADECIMENTOS}

Agradecemos a Universidade Federal de Goiás (UFG), regional Catalão, por nos dar oportunidade de participar do Mestrado em Gestão Organizacional e nos proporcionar iniciação no processo de pesquisa e à ADISGO pelos dados fornecidos.

\section{REFERÊNCIAS}

ADA - American Diabetes Association. (2004) Continuous Subcutaneous Insulin Infusion. Diabetes Care. 27 (1), 110.

Apolinário, D., Silva, I. \& Cardoso M. H. (2011) Construção de Questionários para Avaliação da Qualidade de Vida e Satisfação com o Tratamento com Bomba Infusora de Insulina: Estudo Descritivo e Contributo para a Validação. Dissertação de mestrado, Universidade do Porto, PT, Portugal.

Brod, M., Christensen, T., \& Bushnell, D. (2007) Maximizing the value of validation findings to better understand treatment satisfaction issues for diabetes. Quality of Life Research, 16(6), 1053-1063.

DCCT - Diabetes Control and Complications Trial. (1993) The Diabetes Control and Complications Trial Research Group. The effect of intensive treatment of diabetes on the development and progression of long-term complications in insulin-dependent diabetes mellitus. N Engl J Med. 329:977-86.

Flick, U. (2013) Introdução à metodologia de pesquisa: um guia para iniciantes. Penso Editora.

IDF - International Diabetes Federation (2015) Diabetes Atlas [Internet]. 7ạ ed. Brussels: International Diabetes Federation. Disponível em: http://www.idf.org/diabetesatlas $>$. Acesso em 8-5-16.

Liberatore Jr, R. D. R. \& Damiani, D. (2006) Insulin pump therapy in type 1 diabetes mellitus. Jornal de Pediatria, 82(4), 249-254.

Mala, F. F. R. \& Araújo, L. R. (2003) Uso da bomba de infusão de insulina no tratamento do diabetes mellitus tipo 1:[revisão]. Revista Médica de Minas Gerais, 13(3), 194-199. 
Malerbi, D. A. \& Franco, L. J. (1992) The Brazilian Cooperative Group on the Study of Diabetes Prevalence. Multicenter study of the prevalence of diabetes mellitus and impaired glucose tolerance in the urban Brazilian population aged 30-69 yr. Diabetes Care, 15(11), 1509-16.

Minicucci, W., Figueiredo A., S. T., Araújo, L. R., \& Pimazoni-Netto, A. (2007) O papel da bomba de insulina nas estratégias de tratamento do diabetes. Revista Brasileira de Medicina, (6).

Onkamo, P., Vaananen, S., Karvonen, M., \& Tuomilehto, J. (1999) Worldwide increase in incidence of Type I diabetes \pm the analysis of the data on published incidence trends. Diabetologia, 42 (12), 1395-1403.

SBD - Sociedade Brasileira de Diabetes. (2016) Diretrizes da Sociedade Brasileira de Diabetes. Gerenciamento Eletrônico do Diabetes/ Uso da tecnologia para Melhor Controle Metabólico do Diabetes. São Paulo: AC Farmacêutica.

Schmidt, M. I., Hoffmann, J. F., Diniz, M. D. F. S., Lotufo, P. A., Griep, R. H., Bensenor, I. M. \& Duncan, B. B. (2014) High prevalence of diabetes and intermediate hyperglycemia - The Brazilian longitudinal study of adult health (ELSA-Brasil) Diabetology \& metabolic syndrome, 6(1), 123.

SITEC - I Simpósio Internacional de Tecnologia em Diabetes. (2015) Disponível em: <http: //www.diabetes.org/sitec-2015>. Acesso em 14-5-16. 